\title{
Ankle Intrinsic Stiffness in Subcortical Poststroke Subjects
}

\author{
Andreia S. P. Sousa ${ }^{1}$, Rubim Santos ${ }^{2}$, Augusta Silva ${ }^{1}$ \\ 1 Centro de Estudos de Movimento e Atividade Humana, Área Científica de Fisioterapia, Instituto Politécnico do Porto, Escola \\ Superior de Saúde do Porto, Vila Nova de Gaia, Portugal. ${ }^{2}$ Centro de Estudos de Movimento e Atividade Humana, Área \\ Científica de Física, Escola Superior de Saúde do Porto, Vila Nova de Gaia, Portugal.
}

\begin{abstract}
The authors' purpose was to evaluate bilateral ankle intrinsic stiffness in subcortical poststroke subjects. Ten subcortical poststroke subjects and 10 healthy controls participated in this study. The ankle passive stiffness at 3 different speeds and the electromyographic activity of the soleus, the gastrocnemius, and the tibialis anterior muscles of poststroke contralesional (CONTRA) and ipsilesional (IPSI) limbs and of one limb of healthy subjects were assessed. Ankle electromyographic activity was collected to ensure that reflexive or voluntary muscle activity was not being elicited during the passive movements. A significant interaction was observed between the effects of the limb (IPSI vs. CONTRA vs. control) and ankle position, $F(4,28)=3.285, p=$ .025 , and between the effects of the limb and the velocity of stretch, $F(2,14)=4.209, p=.037$. While increased intrinsic stiffness was observed in the CONTRA limb of poststroke subjects at ankle neutral position when the passive stretch was applied with a velocity of $1 \% \mathrm{~s}(p=.021)$, the IPSI limb of poststroke subjects presented increased stiffness at $20^{\circ}$ of plantar flexion when the stretch was applied with a velocity of $5 \%$ s $(p=.009)$ when compared to healthy group. Subcortical poststroke subjects present increased intrinsic stiffness in both the CONTRA and IPSI limbs in specific ankle amplitudes.
\end{abstract}

Keywords: ankle, contralesional limb, ipsilesional limb, intrinsic stiffness, subcortical stroke

M usculoskeletal stiffness of the ankle joint is recognized as a significant factor contributing to postural stability and locomotion (Clifford \& Wheeless, 2005). It can be decomposed into its intrinsic components arising from the mechanical properties of the joint, passive tissue, active muscle fibers, the dynamic component of viscous damping, and the static component of elastic stiffness (Dietz, Trippel, \& Berger, 1991; Mirbagheri, Barbeau, \& Kearney, 2000; O’Dwyer \& Ada, 1996; Sinkjaer \& Magnussen, 1994) and neural components, including neurophysiological mechanisms and reflex activity (Mirbagheri et al., 2000). The overall dynamic stiffness, and perhaps the relative contributions of its intrinsic and neural components, can be modulated by the nervous system through fusimotor activity (Mirbagheri et al., 2000). Based on this, global stiffness can be altered in dysfunctions which involve one or both of these components.

CNS dysfunctions such as stroke have increased stiffness in the contralesional limb (CONTRA), as a common disorder, together with reduced voluntary muscle activity and postural control (Simpson, Gracies, Yablon, Barbano, \& Brashear, 2009). Increased stiffness has been frequently identified as spasticity related to problems in the central regulation of stretch reflex thresholds (Levin, Selles, Verheul, \& Meijer, 2000). Paradoxically, several studies have failed to find evidence of changes in reflex mechanisms in individuals designated as spastic on clinical examination (Dietz et al., 1991; Lee, Boughton, \& Rymer, 1987; Sinkjaer \& Magnussen, 1994; Toft, Sinkjaer, Andreassen, \& Hansen, 1993). These studies have instead raised the possibility that much of what is considered as spasticity may in fact be caused by changes in intrinsic muscle properties. Several studies have suggested that intrinsic stiffness has profound and consistent effects on chronic poststroke subjects (Ada, Vattanasilp, O'Dwyer, \& Crosbie, 1998; Chung, van Rey, Bai, Roth, \& Zhang, 2004; Dietz et al., 1991; Gracies, 2005; Lamontagne, Malouin, \& Richards, 2000). Various alterations in the musculotendinous structure, such as alterations in muscle fiber size, fiber type distributions, and probably fiber length (Foran, Steinman, Barash, Chambers, \& Lieber, 2005; Lieber, Steinman, Barash, \& Chambers, 2004), together with changes in mechanical (Kamper, Schmit, \& Rymer, 2001; Schmit, Benz, \& Rymer, 2002) and morphological properties of intra- and extracellular materials (Lieber et al., 2004), thixotropic response (Hufschmidt \& Schwaller, 1987), and probably also contractile properties (Hufschmidt \& Mauritz, 1985), may contribute to increased stiffness in poststroke subjects. In line with this, several studies have demonstrated that velocity-dependent changes in stiffness can be a characteristic response of the nonneural structures (Carey \& Burghardt, 1993; Vattanasilp, Ada, \& Crosbie, 2000). Despite the large evidence of the contribution of the intrinsic component of stiffness to increased ankle stiffness in chronic poststroke subjects, no study has considered lesion location (Alibiglou, Rymer, Harvey, \& Mirbagheri, 2008; Gao, Ren, Roth, Harvey, \& Zhang, 2011; Lorentzen et al., 2010; Svantesson, Takahashi, Carlsson, Danielsson, \& Sunnerhagen, 2000) or has included subjects with distinct areas of lesion (O'Dwyer, Ada, \& Neilson, 1996). Given that supraspinal structures can influence intrinsic stiffness by controlling the level of muscle activation (number of actinmyosin crossbridges) existing at an instant (Blanpied \& Smidt, 1992), we consider that a lesion in the territory of the middle cerebral artery, more specifically at the internal capsule, has a high probability of affecting the corticorreticular system (Matsuyama et al., 2004). In this case, we can 
expect changes in the activation of ankle muscles in the CONTRA limb, but also in the ipsilesional limb (IPSI), as a result of ipsilateral disposed systems such as the reticular-spinal system.

While several studies have been dedicated to understand CONTRA limb impairments, fewer studied the IPSI limb. Motor impairments on the IPSI side have been reported in both the upper (Colebatch \& Gandevia, 1989; Desrosiers, Bourbonnais, Bravo, Roy, \& Guay, 1996; Jones, Donaldson, \& Parkin, 1989) and lower extremities (Adams, Gandevia, \& Skuse, 1990; Genthon et al., 2008; Lindmark \& Hamrin, 1995). These motor impairments have been described to result from adaptation to function deficits of the CONTRA limb (Arruin, 2006), as each limb affects the strength of muscle activation and the time-space behavior of the other (Dietz, Zijlstra, \& Duysens, 1994; Stubbs \& Mrachacz-Kersting, 2009). However, the evidence that the CONTRA limb is more influenced by the IPSI limb than the reverse (Sousa, Silva, Santos, Sousa, \& Tavares, 2013) and that bilateral lower limb asymmetry in functional activities is not the primary cause of bilateral postural imbalance (Genthon et al., 2008) sustain the hypothesis that impairments demonstrated in the IPSI limb can result from neuronal damage, depending on the anatomical region of vascular disruption (Matsuyama et al., 2004). Ipsilesional disorders related to impairment of muscles with a predominant postural function, such as the soleus (SOL) muscle, have been demonstrated in poststroke subjects with subcortical injuries located at the internal capsule level (Silva, Sousa, Pinheiro, Ferraz, et al., 2012; Silva Sousa, Pinheiro, Tavares, et al., 2012; Silva, Sousa, Tavares, et al., 2012). Considering this, it can be hypothesized that postural control dysfunction over the IPSI ankle muscles could interfere with ankle intrinsic stiffness regulation. In this sense, it can be hypothesized that increased antagonist coactivation, already demonstrated in the IPSI limb of subcortical poststroke subjects (Silva, Sousa, Tavares, et al., 2012), can be an energetically costly strategy to increase the intrinsic muscle viscoelasticity through an instantaneous process (van der Helm \& Rozendaal, 2000).

In this study we aimed to evaluate bilateral intrinsic stiffness of ankle plantar flexors in subcortical poststroke subjects, and based on the exposed, increased values of intrinsic stiffness were expected in both limbs of poststroke subjects.

\section{Methods}

\section{Subjects}

Ten subjects who had a stroke episode at least six months earlier (four women, six men) and 10 healthy subjects (four women, six men) participated in this study (Table 1). For the poststroke subjects, the mean time between stroke and inclusion in this study was 36 months ( $S D=24.8$ months). All subjects have an ischemic stroke: four of them had an infarction in their left hemisphere, whereas six had an infarction in their right hemisphere. To be included, subjects were required to (a) have a first-ever ischemic stroke involving the middle cerebral artery territory, specifically at the internal capsule, as revealed by computed tomography, resulting in hemiparesis; (b) have a Fugl-Meyer (Assessment of Sensorimotor Recovery After Stroke scale) score in the motor subsection below 34 (Duncan, Propst, \& Nelson, 1983); (3) not have grade 3 score of the Achilles' tendon reflex; (d) present clinical signs of increased muscle tone (with a minimum of 1 in the Modified Ashworth scale) in calf muscles (Bohannon \& Smith, 1987); and (e) have provided written or verbal informed consent to participate in the study. None of the subjects was receiving antispasticity medication.

Subjects were excluded it they (a) had any cognitive deficit that could hinder communication and cooperation (assessed by the Mini-Mental State Examination; Dick et al., 1984), (b) had history of orthopedic or neurological (other than stroke) disorders, known to affect stiffness, (c) had history of stroke involving the brainstem or cerebellar areas, and (d) were taking medication that could affect motor performance. Data of the poststroke group were compared with data obtained from the 10 healthy control subjects. All control subjects were excluded if they presented any neurological and orthopedic disorder. The study was approved by the local ethics committee and implemented according to the Declaration of Helsinki.

\section{Instrumentation}

The electromyographic activity (EMG) of the gastrocnemius medialis (GM), SOL, and tibialis anterior (TA) was monitored using an MP 150 Workstation (Biopac Systems, Inc., Goleta, CA); TD150B steel electrodes with bipolar configuration and $20 \mathrm{~mm}$ between detection surfaces (center to center) and a reference electrode. Skin impedance was measured with an Electrode Impedance Checker (Noraxon USA, Inc., Scottsdale, AZ). The electromyographic signal was processed with Acqknowledge, version 3.9, from Biopac Systems, Inc.

The ankle intrinsic stiffness was assessed using an isokinetic dynamometer, Biodex System 4 Pro (Biodex Medical Systems, Inc., Shirley, NY).

\section{Procedures}

\section{Skin Preparation and Placement of Electrodes}

The skin surface of selected muscles' midbelly and patella was prepared (shaved and then the dead skin cells and nonconductor elements were removed with alcohol and with an abrasive pad) to reduce the electrical resistance to less than $5000 \Omega$. Electrodes were placed according to anatomical references (Table 2) and fixed with adhesive tape, while the reference electrode was placed on the patella. 
TABLE 1. Means and standard deviations for age, height, weight, and ankle range of movement of healthy and poststroke groups.

\begin{tabular}{|c|c|c|c|c|c|}
\hline \multirow[b]{2}{*}{ Variables } & \multicolumn{2}{|c|}{ Poststroke group } & \multicolumn{2}{|c|}{ Healthy group } & \multirow[b]{2}{*}{ p-value } \\
\hline & $M$ & $S D$ & $M$ & $S D$ & \\
\hline Age (years) & 45.88 & 9.43 & 52.75 & 9.21 & .23 \\
\hline Height (m) & 1.72 & 0.10 & 1.66 & 0.11 & .32 \\
\hline Body weight (kg) & 76.25 & 9.22 & 70.05 & 13.57 & .32 \\
\hline \multirow[t]{2}{*}{ Maximal dorsiflexion $\left({ }^{\circ}\right)$} & 0.38 (CONTRA) & 5.95 & 5.38 & 4.78 & .23 \\
\hline & $2.50(I P S I)$ & 3.25 & & & .25 \\
\hline \multirow[t]{2}{*}{ Maximal plantar flexion $\left(^{\circ}\right)$} & $20.63($ CONTRA $)$ & 2.13 & 33.13 & 8.34 & .42 \\
\hline & $22.88(I P S I)$ & 7.10 & & & .51 \\
\hline
\end{tabular}

\section{Data Acquisition}

Subjects were evaluated while seated on an adjustable chair with the knee in full extension. Adjustable straps provided stabilization at chest and waist levels. The thigh was immobilized distally and the foot was kept in the footplate using two adjustable straps. The ankle center of rotation was aligned with the rotational axis of the dynamometer. Three passive dorsiflexions were imposed on both limbs of poststroke subjects and on the right limb of healthy subjects at three different velocities $(5 \% \mathrm{~s}, 1 \%$, and $0.25 \%$ s) from maximum and comfortable plantarflexion to maximum and comfortable dorsiflexion. The velocity of $5 \% \mathrm{~s}$ is slow enough to be used as a subthreshold for evoking a stretch reflex (Hufschmidt \& Mauritz, 1985) and has been used in previous studies (Lamontagne, Malouin, Richards, \& Dumas, 2002). However, as intrinsic stiffness is influenced by proprioceptive information and stiffness modulation by proprioceptive reflexes is more effective in postural tasks (van der Helm \& Rozendaal, 2000), passive stretch with lower velocities was also applied. Pauses of $1 \mathrm{~s}$ separated each passive dorsiflexion, where the ankle was placed at neutral position. After this time, the ankle was moved passively to maximal ankle position. The order of the conditions was randomized. During testing, subjects were instructed to relax all muscles in the lower limb and not to interfere with the passive movements. Before data collection, each subject was given several familiarization trials under each condition. In addition, to allowing subjects to get familiar with the testing procedures, these trials decrease thixotropy (Lakie \& Robson, 1988) and the stress relaxation phenomena (Taylor, Dalton, Seaber, \& Garrett, 1990).

Analog data concerning the angular position and torque from the potentiometer and load cells contained within the dynamometer head were collected at $100 \mathrm{~Hz}$ using an analog-to digital converter and stored on a personal computer for future analysis. Additionally, to ensure that reflexive or voluntary muscle activity was not being elicited during the passive movements, the EMG of the SOL, GM, and TA was acquired with a sample rate of $1000 \mathrm{~Hz}$. The signal was preamplified at the electrode site, passed through a differential amplifier with an adjustable gain setting (12-500 $\mathrm{Hz}$; Common Mode Rejection Ratio: $95 \mathrm{~dB}$ at $60 \mathrm{~Hz}$ and input impedance of $100 \mathrm{M} \Omega$ ), digitized, and then stored in a computer for subsequent analysis with the Acqknowledge software (Biopac Systems, Inc. USA). The gain range was set to 1000 .

\section{Data Processing}

Separate fourth-order polynomial equations relating angular position and torque were constructed for each trial:

$$
y=a x^{4}+b x^{3}+c x^{2}+d x+e
$$

where $Y$ is the gravity corrected torque, $x$ is the angular

TABLE 2. Anatomical references to electrode placement. Electrode locations were confirmed by palpation of the muscular belly with the subject in the test position, being the electrodes placed on the most prominent area.

\begin{tabular}{|c|c|}
\hline Muscle & Electrode placement \\
\hline TA & One third on the line between the tip of the tibia and the tip of the medial malleolus \\
\hline GM & Most prominent bulge of the muscle \\
\hline SOL & $\begin{array}{l}2 \mathrm{~cm} \text { distal to the lower border of the medial gastrocnemius muscle belly and } 2 \mathrm{~cm} \text { medial to the posterior midline of } \\
\text { the leg }\end{array}$ \\
\hline
\end{tabular}




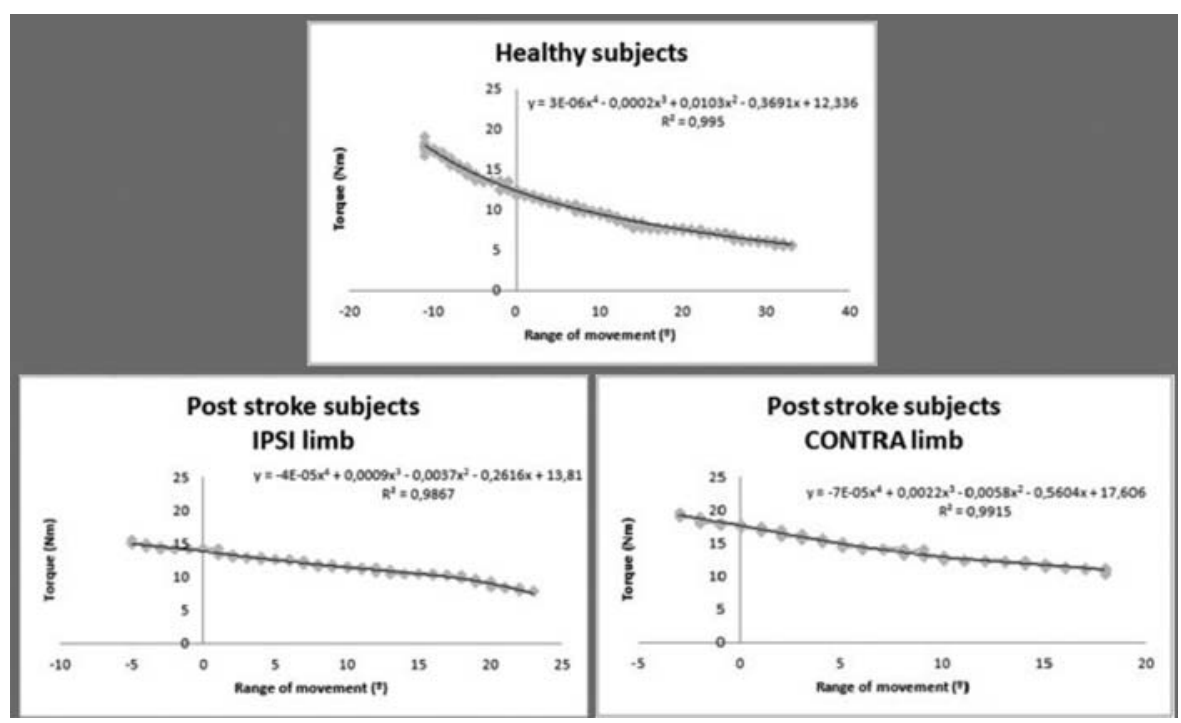

FIGURE 1. Separate fourth-order polynomial equations were constructed relating the angular position and torque data for each trial. Pictured are the original data (rough line), line of the equation (solid) and the $R^{2}$ obtained for a subject of healthy group and a subject of poststroke group when stretch was applied with a velocity of $5 \%$ s. Negative values of range of movement indicate dorsiflexion beyond the neutral position while positive values indicate plantar flexion beyond neutral position.

position, and $a$ through $e$ are constants (Figure 1). High $R^{2}$ values were obtained with fourth-order polynomial equations indicating that data are close to the fitted regression line, which was confirmed by visual inspection (Figure 1). Intrinsic stiffness was calculated at different ankle positions in the sagittal plane, including relative amplitude values but also standard positions important to functional activities. Given the importance of intrinsic stiffness in forward propulsion in poststroke subjects (Lamontagne et al., 2002), the neutral position and the amplitude of plantar flexion required during the late stance phase of gait at low speeds (Nymark, Balmer, Mellis, Lemaire, \& Millar, 2005) were evaluated. In this sense, stiffness values were calculated at maximal and comfortable plantar flexion position (MaxPFP), maximal and comfortable dorsiflexion position (MaxDFP), the mean position between MaxPFP and MaxDFP, neutral position (NP), and $20^{\circ}$ of plantar flexion. To ensure that constant velocity was achieved and maintained throughout all positions analyzed, the first and last $3^{\circ}$ of dorsiflexion were not considered for analysis, to eliminate confounding changes in inertia (Riemann, DeMont, Ryu, \& Lephart, 2001). For each position, the stiffness values were calculated using the first derivative (slope) of the equation $\left(\frac{d y}{d x}=4 a x^{3}+3 b x^{2}+c x+d\right)$, where $\frac{d y}{d x}$ is the stiffness at each of the respective positions. The stiffness represented by taking the slope of the line (Riemann et al., 2001) has been attributed to elastic stiffness at that point in the range of motion (Chesworth \& Vandervoort, 1989; Toft, Espersen, Kalund, Sinkjaer, \& Hornemann, 1989).

The trials with EMG larger than $5 \%$ of the maximum value obtained during passive stretch were rejected to avoid unwanted activations related to voluntary or reflex contractions. The selection of a threshold of $5 \%$ was based on previous studies that used the same criterion to detect muscle activity onset (Polcyn, Lipsitz, Kerrigan, \& Collins, 1998) and on the fact that his method provided a good agreement with visual inspection.

\section{Statistics}

The acquired data were analyzed using the SPSS software from IBM Company (version 20, Armonk, NY). The main effect and interactions between the effects of the limb (IPSI vs. CONTRA), stretch velocities $(5 \% \mathrm{~s}, 1 \% \mathrm{~s}$, and $0.25 \%$ ) and ankle amplitude (MaxPFP vs. MaxDFP vs. mean position between MaxPFP and MaxDFP vs. NP vs. $20^{\circ}$ of plantar flexion) in ankle intrinsic stiffness, as well the differences between groups, were analyzed according to the repeated-measures analysis of variance with betweensubjects factors. Post hoc comparisons were performed with Bonferroni corrections. A significance level of .05 was used for inferential analysis.

\section{Results}

Significant statistical differences were found between healthy and poststroke groups, $F(1,46)=152.27, p<.001$, increased stiffness values were observed in poststroke group (Figure 2). A significant interaction was observed between the effects of the limb (IPSI vs. CONTRA vs. control) and ankle position, $F(4,28)=3.285, p=.025$, and between the effects of the limb and the velocity of stretch, 


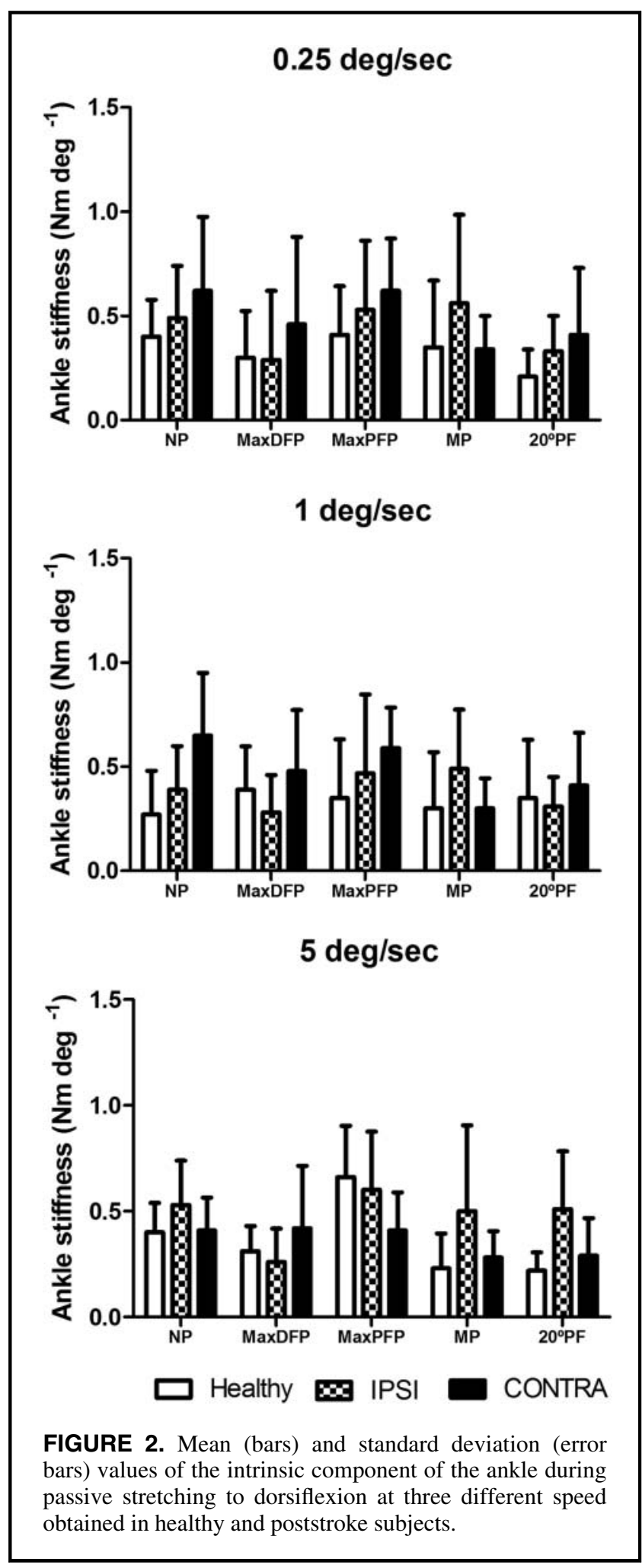

$F(2,14)=4.209, p=.037$. Although the CONTRA limb of poststroke subjects presented more than the double of stiffness values observed in healthy subjects at ankle neutral position when the passive stretch was applied with a velocity of $1 \% \mathrm{~s}(p=.021)$, the IPSI limb presented more than the double of stiffness values observed in healthy subjects at $20^{\circ}$ of plantar flexion when the stretch was applied with a velocity of $5 \% \mathrm{~s}(p=.009)$ when compared with the healthy group (Figures 2 and .).

\section{Discussion}

Intrinsic stiffness properties can be derived from measured torque-angle responses obtained in the absence of significant muscle activation resulting from reflexive activation in response to a sensory stimuli (Gottlieb \& Agarwal, 1978; Sinkjaer, Toft, Andreassen, \& Hornemann, 1988), as judged from EMG recordings. The velocities used to induce the passive displacements were chosen to avoid eliciting stretch reflexes (Hufschmidt \& Mauritz, 1985). Also, a constant velocity protocol was used so that the measured torque response of the limb would be independent of limb inertia, once a steady state in velocity was achieved. The elimination of the first and last $3^{\circ}$ from the analysis guaranteed this assumption. By asking subjects not to intervene with the passive movements we took advantage of the ability to abolish muscle activity through conscious relaxation, thereby eliminating conscious muscle activation as a confounding factor. The relatively few trials in which increased electric activity occurred in our study, coupled with the ability to easily identify and eliminate these instances, support this presumption. Thus, it is reasonable to attribute the resistance measured in response to passive ankle joint displacement into dorsiflexion to the intrinsic stiffness.

Despite a tendency to higher values of plantar flexors' stiffness in the CONTRA and IPSI limbs of poststroke subjects, the differences were more notorious when the ankle was evaluated at standard positions. These results corroborate the results obtained in previous studies (Gao, Grant, Roth, \& Zhang, 2009; Kim, Park, Chon, \& Ohn, 2005; Lorentzen et al., 2010; Vattanasilp et al., 2000) and suggest that increased stiffness obtained in poststroke subjects is largely influenced by reduced ankle range of movement (Table 1). It should be noted that, despite being younger that the subjects included in previous studies, the poststroke subjects of the present study present also bilateral increase of ankle intrinsic stiffness, suggesting that beyond aging (Cenciarini, Loughlin, Sparto, \& Redfern, 2010) subcortical stroke episodes are also a determinant factor for increased intrinsic stiffness.

Changes observed in intrinsic stiffness in the CONTRA ankle can be the result of various alterations in the musculotendinous structure, such as alterations in muscle fiber size, fiber type distributions, and probably fiber length, together with changes in mechanical and morphological properties of intra- and extracellular materials and probably also contractile properties (Foran et al., 2005; Hufschmidt \& Mauritz, 1985; Lieber et al., 2004; Schmit et al., 2002). Considering that the subjects of the present study presented an infarct at the internal capsule, these possible adaptative changes in the musculotendinous structure can result from 


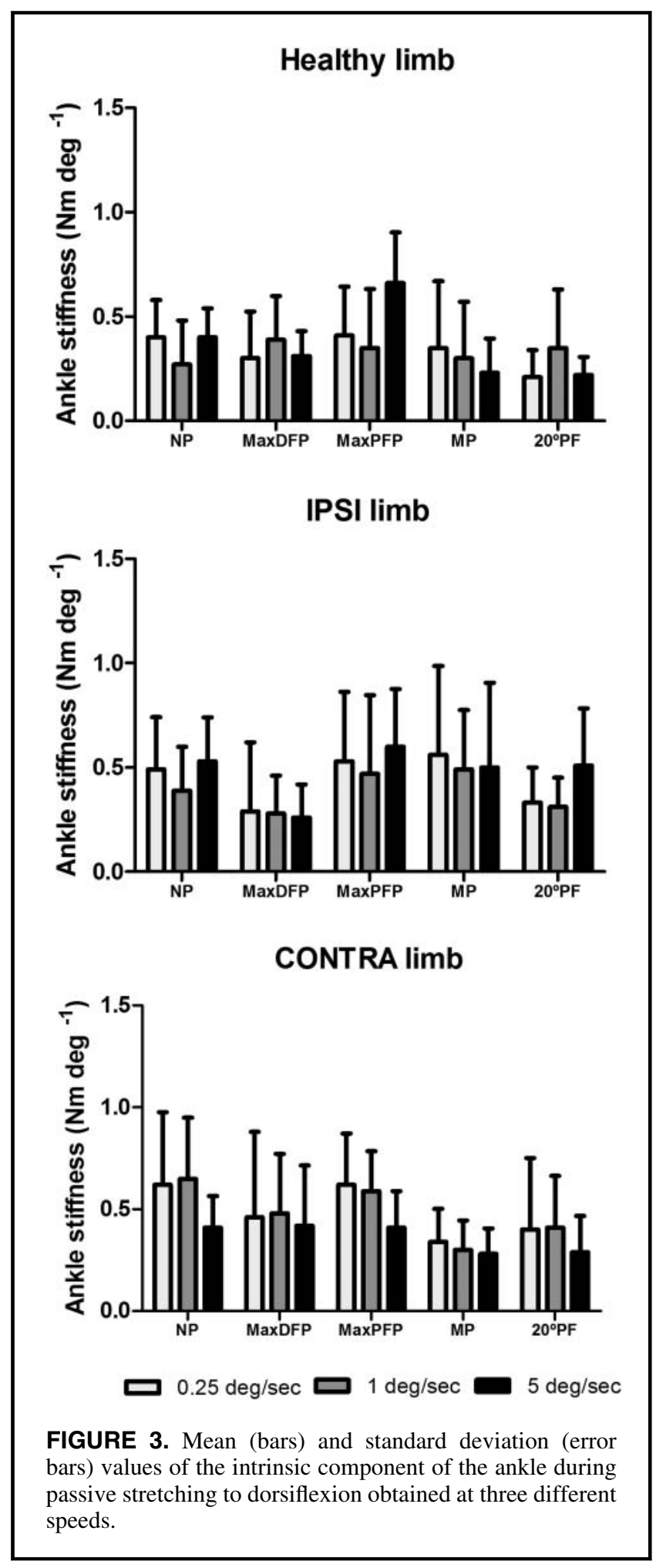

prolonged reduced voluntary muscle activation by the corticospinal system, but also by a deregulation of the vestibular and reticular systems over the SOL tonic activity. A possible damage of corticorreticular system leads to lower activation of motoneurons, as well as of interneurons involved in inhibitory mechanisms from the medial and lateral reticulospinal tract (Brown, 1994) resulting in decreased reciprocal Ia inhibition (Crone, Nielsen, Peterson, Ballegaard, \& Hultborn, 1994), Ib inhibition (Delwaide \& Oliver, 1988), and recurrent inhibition (Mazzocchio \& Rossi, 1997), particularly in antigravity muscles like the SOL. In this case, the vestibular system may assume the function of motor response organization as a more primitive system (Day \& Cole, 2002). It should be also noted that these changes in the musculotendinous structure of plantar flexors are increased by reduced mobility (Roy, Forrester, Macko, \& Krebs, 2013), which would also lead to changes in the mechanical properties of ligaments, tendons, and connective tissue that lead to increased stiffness at and near ankle range of motion limits. Interestingly, the results of the present study indicate that increased ankle stiffness of the CONTRA limb was more pronounced in neutral position compared with the other positions. In these position, poststroke subjects presented more than the double of stiffness values observed in healthy subjects, while in the other positions the ratio observed between healthy subjects and CONTRA limb was between 1.2 (maximal dorsiflexion position) and 1.60 (maximal plantarflexion position). However, values presented in Table 1 indicate that maximal dorsiflexion amplitude were close to the neutral position, suggesting that the major differences between CONTRA limb and controls occurred close to maximal ankle dorsiflexion. It can be argued that higher increased intrinsic stiffness closed to maximal dorsiflexion position enhances the potential capacities of the extrinsic component (Riemann \& Lephart, 2002; van der Helm \& Rozendaal, 2000) during locomotion as stiffer muscles are also believed to transmit loads to muscle spindles more readily, thereby reducing some of the lag time associated with initiation of reflexive activity (van der Helm \& Rozendaal, 2000). However, future studies are required to confirm this hypothesis.

Biomechanical changes in IPSI plantar flexors resulting from postural control failure and reduced mobility can also explain increased stiffness compared to healthy controls. In the IPSI limb higher values of stiffness were observed when ankle plantar flexors were close to their maximum position of muscle shortening. This can result from a higher number of actin-myosin crossbridges in the external amplitude of muscle action related to higher efficiency of ankle plantar flexors' muscle activation during concentric contractions more associated with movement control tasks to the detriment of isometric and excentric contractions more associated with postural control tasks. In fact, recent studies have demonstrated that the IPSI limb presents a dysfunction in ankle muscles' postural control but not in tasks where these muscles are involved in movement control (Silva Sousa, Pinheiro, Ferraz, et al., 2012; Silva, Sousa, Pinheiro, Tavares, et al., 2012; Silva, Sousa, Tavares, et al., 2012; Sousa et al., 2013).

The general increased values of intrinsic stiffness observed in the IPSI limb can also be related to long term changes in the musculotendinous structure's result from a 
strategy used by the CNS, possible through vestibulospinal pathway, to compensate for the corticorreticular dysfunction, typically found in lesions at the internal capsule. In fact, increasing stiffness has been demonstrated to be a strategy used by the CNS to increase stability in unstable postural control conditions (Feldman, 1980; Serres \& Milner, 1991). This can also sustain the fact that differences between the IPSI limb of poststroke subjects and healthy subjects were more notorious when the strech was applied at the higher speed $\left(5^{\circ} / \mathrm{s}\right)$ because higher velocities are associated with higher postural control demand (Cavagna \& Kaneko, 1977).

\section{Conclusion}

The findings obtained in the present study indicate that subcortical poststroke subjects present increased intrinsic stiffness in both CONTRA and IPSI limbs. These results question the most frequent therapeutic approaches that exclude the IPSI limb from therapeutic intervention by considering the IPSI limb as the healthy limb.

\section{References}

Ada, L., Vattanasilp, W., O'Dwyer, N. J., \& Crosbie, J. (1998). Does spasticity contribute to walking dysfunction after stroke? Journal of Neurology, Neurosurgery and Psychiatry, 64, 628-635.

Adams, R. W., Gandevia, S. C., \& Skuse, N. F. (1990). The distribution of muscle weakness in upper motoneuron lesions affecting the lower limb. Brain, 113, 1459-1476.

Alibiglou, L., Rymer, W., Harvey, R., \& Mirbagheri, M. (2008). The relation between Ashworth scores and neuromechanical measurements of spasticity following stroke. Journal of NeuroEngineering and Rehabilitation, 5, 1-14.

Arruin, A. (2006). The effect of asymmetry of posture on anticipatory postural adjustments. Neuroscience Letters, 401(1, 150-153.

Blanpied, P., \& Smidt, G. L. (1992). Human plantarflexor stiffness to multiple single-stretch trials. Journal of Biomechanics, 25, 29-39.

Bohannon, R. and Smith, M. (1987). Interrater reliability of a modified Ashworth scale of muscle spasticity. Physical Therapy, 67(2), 206.

Brown, P. (1994). Pathophysiology of spasticity. Journal of Neurology, Neurosurgery \& Psychiatry, 57, 773-777.

Carey, J. R., \& Burghardt, T. P. (1993). Movement dysfunction following central nervous system lesions: A problem of neurologic or muscular impairment? Physical Therapy, 73, 538-547.

Cavagna, G., \& Kaneko, M. (1977). Mechanical work and efficiency in level walking and running. Journal of Physiology, 268, 467-481.

Cenciarini, M., Loughlin, P. J., Sparto, P. J., \& Redfern, M. S. (2010). Stiffness and damping in postural control increase with age. Biomedical Engineering, IEEE Transactions on, 57, 267-275.

Chesworth, B. M., \& Vandervoort, A. A. (1989). Age and passive ankle stiffness in healthy women. Physical Therapy, 69, 217-224.

Chung, S. G., van Rey, E., Bai, Z., Roth, E. J., \& Zhang, L.-Q. (2004). Biomechanic changes in passive properties of hemiplegic ankles with spastic hypertonia. Archives of Physical Medicine and Rehabilitation, 85, 1638-1646.

Clifford, R., \& Wheeless, M. D. (2005). Wheeless' textbook of orthopaedics. Towson, MD: Data Trace.

Colebatch, J. G., \& Gandevia, S. C. (1989). The distribution of muscular weakness in upper motor neuron lesions affecting the arm. Brain, 112, 749-763.
Crone, C., Nielsen, J. B., Peterson, N., Ballegaard, M., \& Hultborn, H. (1994). Disynaptic reciprocal inhibition of ankle extensors in spastic patients. Brain, 117(Pt5), 1161-1168.

Day, B. L., \& Cole, J. (2002). Vestibular-evoked postural responses in the absence of somatosensory information. Brain, 125, 2081-2088.

Delwaide, P. J., \& Oliver, E. (1988). Short-latency autogenic inhibition (IB inhibition) in human spasticity. Journal of Neurology, Neurosurgery \& Psychiatry, 51, 1546-1550.

Desrosiers, J., Bourbonnais, D., Bravo, G., Roy, P.-M., \& Guay, M. (1996). Performance of the 'unaffected' upper extremity of elderly stroke patients. Stroke, 27, 1564-1570.

Dick, J. P., Guiloff, R. J., et al. (1984). Mini-mental state examination in neurological patients. Journal of Neurology, Neurosurgery and Psychiatry, 47(5), 496-499.

Dietz, V., Trippel, M., \& Berger, W. (1991). Reflex activity and muscle tone during elbow movements in patients with spastic paresis. Annals of Neurology, 30, 797-779.

Dietz, V., Zijlstra, W., \& Duysens, J. (1994). Human neuronal interlimb coordination during split-belt locomotion. Experimental Brain Research, 101, 513-520.

Duncan, P. W., Propst, M., \& Nelson, S. G. (1983). Reliability of the fugl-meyer assessment of sensorimotor recovery following cerebrovascular accident. Physical Therapy, 63, 1606-1610.

Feldman, A. (1980). Superposition of motor programs: 1. Rhythmic forearm flexion in man. Neuroscience, 5, 81-90.

Foran, J. R. H., Steinman, S., Barash, I., Chambers, H. G., \& Lieber, R. L. (2005). Structural and mechanical alterations in spastic skeletal muscle. Developmental Medicine \& Child Neurology, 47, 713-717.

Gao, F., Grant, T. H., Roth, E. J., \& Zhang, L.-Q. (2009). Changes in passive mechanical properties of the gastrocnemius muscle at the muscle fascicle and joint levels in stroke survivors. Archives of Physical Medicine and Rehabilitation, 90, 819-826.

Gao, F., Ren, Y., Roth, E. J., Harvey, R., \& Zhang, L.-Q. (2011). Effects of repeated ankle stretching on calf muscle-tendon and ankle biomechanical properties in stroke survivors. Clinical Biomechanics, 26, 516-522.

Genthon, N., Rougier, P., Gissot, A.-S., Froger, J., Pélissier, J., \& Pérennou, D. (2008). Contribution of each lower limb to upright standing in stroke patients. Stroke, 39, 1793-1799.

Gottlieb, G. L., \& Agarwal, G. C. (1978). Dependence of human ankle compliance on joint angle. Journal of Biomechanics, 11, 177-181.

Gracies, J.-M. (2005). Pathophysiology of spastic paresis. I: Paresis and soft tissue changes. Muscle \& Nerve, 31, 535-551.

Hufschmidt, A., \& Mauritz, K. H. (1985). Chronic transformation of muscle in spasticity: A peripheral contribution to increased tone. Journal of Neurology, Neurosurgery \& Psychiatry, 48, 676-685.

Hufschmidt, A., \& Schwaller, I. (1987). Short-range elasticity and resting tension of relaxed human lower leg muscles. Journal of Physiology, 391, 451-465.

Jones, R. D., Donaldson, I. M., \& Parkin, P. J. (1989). Impairment and recovery of ipsilateral sensory-motor function following unilateral cerebral infarction. Brain, 112, 113-132.

Kamper, D. G., Schmit, B. D., \& Rymer, W. Z. (2001). Effect of muscle biomechanics on the quantification of spasticity. Annals of Biomedical Engineering, 29, 1122-1134.

Kim, D. Y., Park, C., Chon, J. S., \& Ohn, S. H. (2005). Biomechanical assessment with electromyography of post-stroke ankle plantar flexor spasticity. Yonsei Medical Journal, 46, 546-554.

Lakie, M., \& Robson, L. G. (1988). Thixotropic changes in human muscle stiffness and the effects of fatigue. Quaterly Journal of Experimental Physiology, 73, 487-500.

Lamontagne, A., Malouin, F., \& Richards, C. L. (2000). Contribution of passive stiffness to ankle plantarflexor moment during 
gait after stroke. Archives of Physical Medicine and Rehabilitation, 81, 351-358.

Lamontagne, A., Malouin, F., Richards, C. L., \& Dumas, F. (2002). Mechanisms of disturbed motor control in ankle weakness during gait after stroke. Gait \& Posture, 15, 244-255.

Lee, W. A., Boughton, A., \& Rymer, W. Z. (1987). Absence of stretch reflex gain enhancement in voluntarily activated spastic muscle. Experimental Neurology, 98, 317-335.

Levin, M. F., Selles, R. W., Verheul, M. H. G., \& Meijer, O. G. (2000). Deficits in the coordination of agonist and antagonist muscles in stroke patients: Implications for normal motor control. Brain Research, 853, 352-369.

Lieber, R. L., Steinman, S., Barash, I. A., \& Chambers, H. (2004). Structural and functional changes in spastic skeletal muscle. Muscle \& Nerve, 29, 615-627.

Lindmark, B., \& Hamrin, E. (1995). Relation between gait speed, knee muscle torque and motor scores in post-stroke patients. Scandinavian Journal of Caring Sciences, 9, 195-202.

Lorentzen, J., Grey, M. J., Crone, C., Mazevet, D., Biering-Sørensen, F., \& Nielsen, J. B. (2010). Distinguishing active from passive components of ankle plantar flexor stiffness in stroke, spinal cord injury and multiple sclerosis. Clinical Neurophysiology, 121, 1939-1951.

Matsuyama, K., Mori, F., Nakajima, K., Drew, T., Aoki, M., \& Mori, S. (2004). Locomotor role of the corticoreticular-reticulospinal-spinal interneuronal system. In D. G. S. Shigemi Mori \& W. Mario (Eds.), Progress in Brain Research (pp. 239-249). London: Elsevier.

Mazzocchio, R., \& Rossi, A. (1997). A method for potentiating Renshaw cell activity in humans. Brain Research Protocols, 2, 53-58.

Mirbagheri, M., Barbeau, H., \& Kearney, R. E. (2000). Intrinsic and reflex contributions to human ankle stiffness: Variation with activation level and position. Experimental Brain Research, 135, 423-436.

Nymark, J. R., Balmer, S. J., Mellis, E. H., Lemaire, E. D., \& Millar, S. (2005). Electromyographic and kinematic nondisabled gait differences at extremely slow overgroung and treadmill walking speeds. Journal of Rehabilitation Research \& Development, 42, 523-534.

O’Dwyer, N. J., \& Ada, L. (1996). Reflex hyperexcitability and muscle contracture in relation to spastic hypertonia. Current Opinion in Neurology, 9, 451-455.

O'Dwyer, N. J., Ada, L., \& Neilson, P. D. (1996). Spasticity and muscle contracture following stroke. Brain, 119, 1737-1749.

Polcyn, A. F., Lipsitz, L. A., Kerrigan, D. C., \& Collins, J. J. (1998). Age-related changes in the initiation of gait: Degradation of central mechanisms for momentum generation. Archives of Physical Medicine and Rehabilitation, 79, 1582-1589.

Riemann, B. L., \& Lephart, S. M. (2002). The sensoriomotor system, part II: The role of proprioception in motor controlo and fucntional stability. Journal of Athletic Training, 37, 80-84.

Riemann, B. R., DeMont, R. G., Ryu, K., \& Lephart, S. M. (2001). The effects of sex, joint angle, and the gastrocnemius muscle on passive ankle joint complex stiffness. Journal of Athletic Training, 36, 369-377.

Roy, A., Forrester, L. W., Macko, R. F., \& Krebs, H. I. (2013). Changes in passive ankle stiffness and its effects on gait function in people with chronic stroke. Journal of Rehabilitation Research \& Development, 50, 555-572.

Schmit, B., Benz, E., \& Rymer, W. Z. (2002). Afferent mechanisms for the reflex response to imposed ankle movement in chronic spinal cord injury. Experimental Brain Research, 145, 40-49.
Serres, S. J., \& Milner, T. E. (1991). Wrist muscle activation patterns and stiffness associated with stable and unstable mechanical loads. Experimental Brain Research, $86,451-458$.

Silva, A., Sousa, A. S. P., Pinheiro, R., Ferraz, J., Tavares, J. M. R. S., Santos, R., \& Sousa, F. (2012). Activation timing of soleus and tibialis anterior muscles during sit-to-stand and stand-to-sit in post-stroke vs healthy subjects. Somatossensory and Motor Research, 30, 48-85.

Silva, A., Sousa, A. S. P., Pinheiro, R., Tavares, J. M. R. S., Santos, R., \& Sousa, F. (2012). Soleus activity in post-stroke subjects: Movement sequence from standing to sitting. Somatossensory and Motor Research, 29, 71-76.

Silva, A., Sousa, A. S. P., Tavares, J., Tinoco, A., Santos, R., \& Sousa, F. (2012). Ankle dynamic in stroke patients. Agonist vs antagonist muscle relations. Somatosensory and Motor Research, 29, 111-116.

Simpson, D. M., Gracies, J. M., Yablon, S. A., Barbano, R., \& Brashear, A. (2009). Botulinum neurotoxin versus tizanidine in upper limb spasticity: A placebo-controlled study. Journal of Neurology, Neurosurgery and Psychiatry, 80, 380-385.

Sinkjaer, T., \& Magnussen, I. (1994). Passive, intrinsic and reflexmediated stiffness in the ankle extensors of hemiparetic patients. Brain, 117(Pt 2), 355-363.

Sinkjaer, T., Toft, E., Andreassen, S., \& Hornemann, B. C. (1988). Muscle stiffness in human ankle dorsiflexors: Intrinsic and reflex components. Journal of Neurophysiology, 60, 1110 1121.

Sousa, A. S. P., Silva, A., Santos, R., Sousa, F., \& Tavares, J. M. R. S. (2013). Interlimb coordination during the stance phase of gait in subjects with stroke. Archives of Physical Medicine and Rehabilitation, 94, 2515-2522.

Stubbs, P. W., \& Mrachacz-Kersting, N. (2009). Short-latency crossed inhibitory responses in the human soleus muscle. Journal of Neurophysiology, 102, 3596-3605.

Svantesson, U., Takahashi, H., Carlsson, U., Danielsson, A., \& Sunnerhagen, K. S. (2000). Muscle and tendon stiffness in patients with upper motor neuron lesion following a stroke. European Journal of Applied Physiology, 82, 275-279.

Taylor, D. C., Dalton, J. D., Seaber, A. V., \& Garrett, W. E. (1990). Viscoelastic properties of muscle-tendon units. The biomechanical effects of stretching. American Journal of Sports Medicine, 18, 300-309.

Toft, E., Espersen, G. T., Kalund, S., Sinkjaer, T., \& Hornemann, B. C. (1989). Passive tension of the ankle before and after stretching. American Journal of Sports Medicine, 17, 489-494.

Toft, E., Sinkjaer, T., Andreassen, S., \& Hansen, H. J. (1993). Stretch responses to ankle rotation in multiple sclerosis patients with spasticity. Electroencephalography and Clinical Neurophysiology, 89, 311-318.

van der Helm, F. T., \& Rozendaal, L. (2000). Musculoskeletal Systems with Intrinsic and Proprioceptive Feedback. In J. Winters \& P. Crago (Eds.), Biomechanics and neural control of posture and movement (pp. 164-174). New York, NY: Springer.

Vattanasilp, W., Ada, L., \& Crosbie, J. (2000). Contribution of thixotropy, spasticity, and contracture to ankle stiffness after stroke. Journal of Neurology, Neurosurgery and Psychiartry, 69, 34-39. 